\title{
Whitehead, Quantum Mechanics and Local Realism
}

\section{Leemon McHenry}

LEEMON McHENRY teaches philosophy at Loyola Marymount University, 7900 Loyola Boulevard, Los Angeles, CA 90045 and California State University, 18111 Nordhoff Street, Northridge, CA 91330. E-mail: Imchenry@lmumail.lmu.edu

In his book, Nature Loves to Hide, Shimon Malin provides a wonderfully lucid and fascinating account of the development of quantum mechanics in the twentieth century and argues that the baffling puzzles of the quantum world can be understood in terms of Whitehead's process metaphysics. ${ }^{1}$ Malin thus joins an ever-growing number of physicists who see in Whitehead's theories a useful framework for understanding contemporary physics, including for example, Henry Stapp, Timothy Eastman, Lawrence Fagg, Basil Hiley and David Finkelstein.

Nature Loves to Hide is divided into three parts: "The Quandary," "From a Universe of Objects to a Universe of Experiences," and "Physics and the One." Malin also includes three appendices on the relativity of simultaneity, the proof of Bell's inequalities, and interpretations of quantum theory (i.e., quantum ontologies).

"The Quandary" examines the debate between Niels Bohr and Albert Einstein regarding the completeness of quantum mechanics. Einstein believed that quantum mechanics violates both local realism and determinism and therefore fails as a complete, fundamental theory of nature while Bohr held that quantum mechanics was complete regardless of its violations. Bohr's radical thesis was that a single model cannot adequately describe the atomic and subatomic realms. His complementarity framework of wave/particle applies to different conditions, but even here Bohr is not describing nature itself, but rather what we can know about nature. Malin distinguishes between Einstein's ontic approach and Bohr's epistemic approach (39). The latter, which became the orthodox "Copenhagen Interpretation," is often described as pragmatic or instrumentalist since quantum theory is to be viewed as merely a set of rules for calculating correlations among observations. As the Einstein-Bohr controversy continued through their disciples, John Bell demonstrated in 1964 that the issue was not merely philosophical but rather scientific when his theorem led to an experiment that proved nature violates local realism. At this round, Bohr wins, Einstein loses. Malin understands "the quandary" to be the fact that local realism must be abandoned even if it is the "eminently reasonable" world-view to which we continue to cling. Physics (post Bell's theorem) has suffered a type of "schizophrenia" since physicists adhere to Bohr's view as the orthodox interpretation of quantum mechanics, yet, at the same time, rely on Einstein's paradigm at some fundamental level. Malin's 
attempt to resolve the quandary begins with "a close look at the assumption of realism" (87). He contends that both Whitehead and Erwin Schrödinger provide support for the conclusion that realism must be replaced with a theory about the universe as constituted of throbs of experience.

Part Two, "From a Universe of Objects to a Universe of Experiences" attempts to construct such a theory by first examining the collapse of a quantum state (or wave function) and then by providing the ontic interpretation of such states in terms of Whitehead's actual occasions. Quantum entities, such as electrons, exist as fields of potentialities. Once a measurement takes place the potential becomes actual. This is the collapse; that is, the probability distribution has "collapsed" to a single location. Malin shows how this process is atemporal because nothing is actually contributed to the physical world until the collapse occurs. The quantum entity is created in the measurement process once the potential becomes actual. Prior to its actuality there is no entity to speak of, but once the process is complete, an event in physical space and time occurs. This is Heisenberg's contribution. In Physics and Philosophy, he writes:

The transition from the "possible" to the "actual" takes place during the act of observation. If we want to describe what happens in an atomic event, we have to realize that the word "happens" can apply only to the observation, not to the state of affairs between two observations. It applies to the physical, not the psychical act of observation, and we may say that the transition from the "possible" to the "actual" takes place as soon as the interaction of the object with the measuring device, and thereby with the rest of the world, has come into play; it is not connected with the act of registration of the result by the mind of the observer. (54-55)

This is where Whitehead enters the picture. Malin thinks that: "The hallmark of a successful new paradigm is the easy resolution of problems that are, from the point of view of the old paradigm, puzzling and disturbing" (176). He says that there cannot be complete agreement between Whitehead's philosophy and quantum mechanics since the latter excludes experiences from its domain of inquiry. Nonetheless the objective aspects of Whitehead's system accord with findings of quantum mechanics. As he puts the point, "Features that are strange and puzzling when we follow our habitual ways of thinking become simple and natural within a Whiteheadian way of thinking” (177). Although Malin doesn't mention the theories by name, he clearly has in mind Whitehead's epochal theory of becoming and the model of the actual occasion as an atemporal process of concrescence. He also finds a striking affinity between Paul Dirac's view that the collapse occurs as a result of nature making a choice and Whitehead's theory that an actual occasion creates itself by prehensions of its immediate past.

In Part Three, "Physics and the One," Malin examines further the notion of choice in nature by exploring the hierarchy of being in Plato and the neoplatonist, Plotinus. But his main problem is subjectivity. In so far as science excludes the Subject of Cognizance from its inquiries, it has removed life from nature. In this way a fully developed Theory of Everything is beyond reach. He therefore 
contends that "the integration of the objective and subjective domains within the context of the scientific endeavor will be the next decisive step in the evolution of science" (Nature 230, his emphasis).

Since readers of this journal will be especially interested in the Whiteheadian framework by which Malin interprets quantum mechanics, I will focus mainly on some problems in the parts of the book devoted to Whitehead. Malin gets the gist of Whitehead's metaphysics while not involving himself in the detailed technicalities. But it is in such details that one finds some differences with the view of Whitehead that Malin espouses.

It is well known that Whitehead formulated his event ontology and later his atomistic process metaphysics with the quantum theory in mind. Yet it was the early theories of Planck and Bohr and not the later developments of Heisenberg and Schrödinger that influenced his thinking. ${ }^{2}$

So, it is especially surprising that Malin sees Whitehead as the visionary who provides a coherent paradigm for understanding these puzzling developments. As to the central issue of local realism, it is not exactly clear how Whitehead provides the solution. Within the context of the Bohr-Einstein debate, local realism asserts: (1) an event in one place can affect another (subject to limitations imposed by the speed of light), and (2) that physical objects exist independently of consciousness. Malin says in order to cure physics of its "schizophrenia" realism must be replaced with a theory of the universe as constituted of throbs of experience. But just exactly how is it that Whitehead's theory replaces local realism? As for (1), Malin distinguishes (following Henry Stapp) between "signals" and "influences" $(83,97)$. The former cannot propagate faster than light. This applies within the limited realm of classical physics and Special Relativity. However, within the quantum domain, influences can. A measurement performed on one particle determines instantly the corresponding quantity of another particle. As for (2), Malin says: "When we accept the realist position, we feel that we cannot deny the "fact" that objects exist "from their own side," independently of consciousness. On the other hand, we cannot deny that we do have experiences; i.e., we cannot deny the existence of mind" (97). The problem is based on the mistaken conceptual framework of the mind/matter dichotomy repudiated by Whitehead's fallacy of misplaced concreteness. Malin thinks that Whitehead demonstrated that realism is not a fundamental characteristic of reality since experiences, not objects, are concrete. Realism, for Malin, is an abstraction of thought, of the duality of mind and matter, subject and object, measurer and measured, that creates problem. ${ }^{3}$

As he makes his central point, he writes: "Whitehead's realization of 'the fallacy of misplaced concreteness' and Schrödinger's analysis of 'the principle of objectivation' both lead to the conclusion that so-called objects are mere mental constructs. It follows that 'the real world,' in itself, is not a collection of objects" (109). 
It may be simply a matter of clarification of terms, but Whitehead is not an opponent of realism. To argue, as he does, that experience is the primary reality in the most basic units of process does not mean that his view collapses into some version of subjective Idealism. That is, he never abandoned the idea of an objective, physical nature independent of human perceptions. Whitehead begins with a common world in which we find ourselves, other minds and other entities that exist in their own right (Science 90-91). But the objective world or "stubborn fact" is construed as the settled, immediate past of actualities in the process of self-creation. The whole subject-object relation is reformulated as a temporal relation of present to past. Hartshorne once called this position "realistic-idealism" in articulating the asymmetries of process. The past is independent of the present, but the present is dependent on the past. ${ }^{4}$

It is clear that Whitehead's concept of the actual occasion replaces the ultimate reality of the mind/matter distinction, but it is less clear how this helps us understand the measurement problem in quantum mechanics. First, the measurement apparatus whereby quantum events are observed is already a highly abstract set up. The physical process isolated for the sake of what physicists dub a 'measurement' does not quite conform to Whitehead's view of concrete reality. Second, sub-atomic particles such as electrons and photons are not to be equated with actual occasions or basic throbs of experience. (Malin's discussion conflates the two at several points, e.g., Nature 184). For Whitehead, sub-atomic particles are "societies." What the physicist observes, namely, the effects of subatomic particles in the electromagnetic field, is multiple interactions of events with an electromagnetic character. As he says, "The notion of physical energy, which is at the base of physics, must then be conceived as an abstraction from the complex energy, emotional and purposeful, inherent in the subjective form of the final synthesis in which each occasion completes itself" (Adventures 186). Third, and closely related to my second point, the idea that nature is alive, for Whitehead, applies at the most basic level of a universe made up of creative actualities and at various points within the extensive continuum. Sentience applies at some levels of social order and not at others (Process 103). Quantum events would not count as sentient. The societies of subatomic particles contain actual occasions with a dominance of the physical poles, but there is nothing that would give us the kind of selective choice that Malin attributes to this level of nature.

Oddly enough, Malin never uses the terms "panpsychism," or "pan-experientialism" to describe the position he is advancing, but his emphasis on what he calls the "aliveness of nature" and his repudiation of the lifeless mechanistic views of physics makes it clear that he embraces some version of these doctrines. (His chapter 12 is entitled "Nature Alive" which is presumably an allusion to the chapter of the same title in Whitehead's Nature and Life reprinted in Modes of Thought.)

Quantum mechanics is an amazingly successful theory empirically but the orthodox version developed in such a haphazard manner that it has become baf- 
flingly incomprehensible and wildly implausible. Since Bohr, Heisenberg, Born, Dirac and others did not develop a consistent model that could do justice to both wave and particle behavior of quantum systems, they developed the theory in such a way that it is restricted to predicting the outcome of performing measurements on quantum systems. This has the puzzling consequence of viewing the occurrence of events in nature as dependent upon our presence, and specifically our activities as physicists who perform the measurements (Comprehensibility 234). Given Whitehead's view of the extensive continuum-electrons, protons, atoms, molecules, and so on-entities exist objectively regardless of whether measurements are made. Instead of treating quantum mechanics as some special, isolated theory of the micro-world, he attempted to develop a theory of the world in which all sciences are united.

Malin, I think, correctly sees an affinity between certain aspects of quantum phenomena and Whitehead's epochal theory of becoming. Whitehead tells us in Science and the Modern World that it is equally possible to arrive at his organic conception of the world from psychology on the one hand and from mathematical physics on the other (152). The former involves the development of gestalt theories and the introspective psychology that led to the notion of the specious present (Science 73, 104; Concept 56). The latter involves the dematerialization of nature implied by advancing physics in the nineteenth and twentieth centuries. Energy becomes fundamental thereby displacing matter from its position in classical physics. Leaving aside the problem of measurement and local realism for the moment, one sees in Whitehead's epochal theory of becoming some of the basic features of quantum phenomena. In one passage of Science and the Modern World devoted to the quantum theory, he identifies the main revision of physical concepts demanded by the quantum theory. He says: "some theory of discontinuous existence is required. What is asked from such a theory, is that an orbit of an electron can be regarded as a series of detached positions, and not as a continuous line" (135). The doctrine of the specious present in psychology and the superposition of an electron in physics provided the empirical evidence for the more general metaphysical doctrine of epochal becoming. Continuity of space-time arises from the extensive relations realized at a more basic level of discontinuous existence. Moreover, in accordance with Planck's discovery that minimal packets of energy, or quanta, that cannot be subdivided, an actual occasion becomes all at once as an epochal duration and not in bits and pieces. Thus quantum theory and the epochal theory of becoming solve Zeno's paradoxes in the same way. Continuity is rejected as a basic feature of the units of becoming, but in the succession of the units of becoming what becomes is continuity. In Whitehead's theory, this quasi-temporal realm is called "the genetic process" (Process 283). ${ }^{5}$ Though the act of becoming in the genetic process is not continuous or extensive, it delivers to the world a definite temporal quantum (Process 69).

As mentioned above, Malin is not alone among physicists who see in Whitehead's metaphysics a useful guide for understanding certain aspects of quantum 
mechanics or the possibility of developing a more unified and comprehensive theory, i.e., one that is not treated in isolation from the rest of physical theory. But the question remains as to whether Malin has read more into Whitehead's views than is really there. This is not to say that Whitehead's theory cannot be modified in light of advancing physics. However, the rejection of realism in order to develop a more comprehensive quantum theory is not part of Whitehead's project. In this regard, Whitehead stands firmly with Einstein who complained so vigorously to Schrödinger that Bohr, Heisenberg et al. "simply do not see the sort of risky game they are playing with reality-reality as something independent of what is experimentally established" (Przibram 39). As the pursuit continues for Grand Unification Theories and a Theory of Everything, one of the most important links is the unification of quantum mechanics with general relativity. Something will have to give in the grand synthesis if it is ever to be achieved. I would hazard the guess that the peculiarities of the Bohr-Heisenberg model of quantum mechanics and specifically the antirealist stance will not survive. This being the case, Einstein might very well win the day.

Malin's book has great value for his clear statement of the problem, that is, the quandary; the solution of the problem he advances, however, is flawed, at least in so far as it is meant to be in consonance with Whitehead's philosophy.

\section{Notes}

1. I wish to thank Nicholas Maxwell for helpful discussions on this topic over many years and for his input on an earlier draft of this paper. Lewis S. Ford also provided helpful comments.

2. See, Folse 261. Folse, by the way, rejects the view that Bohr had merely an epistemic or instrumentalist view of quantum theory as is commonly believed. He writes: "Bohr argues that the complementarity of particle and wave "pictures" is a consequence of the fact that observation must be theoretically represented as an interaction in which one of the interacting physical systems is understood to be the real object which quantum theory attempts to describe" (262).

3. "Realism" is a term that typically denotes a theory of the world rather than a feature of the world. Malin seems to use the terms "realism" and "objectivity" synonymously.

4. In this connection, Henry Strapp has argued that Whitehead provides a theoretical framework for quantum mechanics that accords with the ontological approach of Heisenberg and with Einstein's view that "physical theories should refer normally to the objective physical situation, rather than our knowledge of that system" (1).

5. I use the term "quasi-temporal" in place of Malin's term "atemporal" since the latter suggests something closer to the abstract status of eternal objects in Whitehead's system. Although the conscrescence of the occasion does not occur in physical time, it is not quite accurate to imply that the genetic process is completely devoid of temporality. 


\section{Works Cited}

Folse, Henry J. "Complementarity, Bell's Theorem, and the Framework of Process Metaphysics." Process Studies 11 (1981): 259-73.

Heisenberg, Werner. Physics and Philosophy: The Revolution in Modern Science. New York: Harper and Brothers Publishers, 1958.

Malin, Shimon. Nature Loves to Hide: Quantum Physics and the Nature of Reality, a Western Perspective. New York: Oxford UP, 2000.

Maxwell, Nicholas. The Comprehensibility of the Universe: A New Conception of Science. Oxford: Clarendon Press, 1998.

Przibram, K., ed. Albert Einstein: Letters on Wave Mechanics. New York: Philosophical Library, 1986.

Stapp, Henry P. "Whiteheadian Approach to Quantum Theory and the Generalzed Bell's Theorem.” Foundations of Physics 9 (1979): 1-25.

Whitehead, Alfred North. Process and Reality: An Essay in Cosmology. 1929. Corrected Edition. Ed. David Ray Griffin and Donald W. Sherburne. New York: Free Press, 1978.

—. Science and the Modern World . New York: Free Press, 1967.

- The Concept of Nature. Cambridge: Cambridge UP, 1920. 


\section{Whitehead's Philosophy and Quantum Physics: A Defense of Nature Loves to Hide}

Shimon Malin

SHIMON MALIN teaches physics at Colgate University, 13 Oak Dr., Hamilton, New York, 13346. E-mail: smalin@mail.colgate.edu

Leemon McHenry's thoughtful and thorough discussion of my book, Nature Loves to Hide, can be divided into two parts. The first part is a summary of the book and the second contains critical comments. In this essay I will address the issues raised in the second part in the order of their presentation.

McHenry's first critical comment is that, in view of the fact that Whitehead was not influenced by the mature quantum theory of Heisenberg and Schrödinger, "it is especially surprising that Malin sees Whitehead as a visionary who provides a coherent paradigm for understanding these puzzling developments." For me this is precisely what is so impressive about Whitehead's system. Although it was not created with the mature quantum theory in mind, it does provide this theory with a metaphysical foundation that fits it so well.

The second and major issue raised by McHenry is the question of whether or not Whitehead's philosophy transcends realism. He writes: "It may be simply a matter of clarification of terms, but Whitehead is not an opponent of realism. To argue, as he does, that experience is the primary reality in the most basic units of process does not mean that this view collapses into some version of subjective Idealism. That is, he never abandoned the idea of an objective, physical nature independent of human perceptions." Well, I agree with the thrust of this statement, and it is, indeed, a question of clarification of terms. My definition of "realism" is as follows: "Realism" is "the belief that the physical world consists of objects which exist 'from their own side,' i.e., independently of consciousness" (Nature 1). By the term "objects" I mean "inanimate chunks of matter." Since Whitehead's actual occasions are not "objects" in this sense, his philosophy is not a kind of "realism" in my sense of the term "realism." Indeed, the thrust of the book is certainly not to indicate that the emergent world view is "some version of subjective Idealism." The emergent world view presented in the book is fully in accord with "the idea of an objective, physical nature independent of human perceptions."

Philosophically, my definition of "realism" may be criticized as being too narrow. This is, however, the kind of realism adhered to tacitly or even explicitly by most scientists.

As for my quoted statement, "Whitehead's realization of 'the fallacy of misplaced concreteness' and Schrödinger's analysis of 'the principle of objectivation' both lead to the conclusion that so-called objects are mere mental constructs," it merely states that the objects we see all around us are our mental constructs; 
it does not mean to imply that the building blocks of reality are our mental constructs.

Having made my position clear, let me add that McHenry's misreading of my position is entirely understandable, since I used the term "realism" in reference to the building blocks of reality and in reference to the nature of our perceptions interchangeably, without making the proper distinction.

With all this in view, I believe that McHenry's question, "just exactly how it is that Whitehead's theory replaces local realism" (italics are his), is answered in detail in Chapter 15 of the book (and see also Chap. 16, Sec. 6).

The next issue is the question of how Whitehead's concept of actual occasions helps us to "understand the measurement problem in quantum mechanics." This question is answered in detail in Chapters 10,13 and 15. McHenry presents three objections to my analysis; let us consider them in turn.

McHenry writes: "First, the measurement apparatus whereby quantum events are observed is already a highly abstract set up. The physical process isolated for the sake of what physicists dub 'measurement' does not quite conform to Whitehead's view of concrete reality." Well, here too it is a matter of clarification of terms. What I mean by the term "quantum measurement" is not necessarily an interaction between a quantum system and a measuring apparatus that was set up by a scientist. As the repeatedly used example of the electron impinging on a TV screen indicates, the term "quantum measurement" is used in a generalized sense, covering any interaction between a quantum system and an everyday size system that can bring about an observable change in the latter. I must admit, however, that this usage of the term "quantum measurement" is far from obvious. I should have stated it explicitly in the book.

McHenry's second objection is that "sub-atomic particles such as electrons and photons are not to be equated with actual occasions or basic throbs of experience (Malin's discussion conflates these two at several points. e.g., Malin, Nature 184)." My response to this point hinges on the following general comment: in invoking "a Whiteheadian approach" in relation to quantum physics, I do not have in mind Whitehead's philosophical system as stated by Whitehead, in all its details. What I have in mind, rather, is (to use Abner Shimony's words) a discussion of "the possibility of a modified philosophy of organism, which would preserve Whitehead's essential ideas while according with the discoveries of modern physics" (Search 292). Whitehead's statements about electrons and photons are irrelevant from this point of view, since he wrote them from the perspective of the early quantum theory of the first two decades of the $20^{\text {th }}$ century, a perspective that was later superceded by the mature quantum theory of the 1920s. (The same comment applies to Whitehead's statements about the orbits of electrons which are mentioned later in the discussion.) My discussion of the elementary quantum events associated with electrons in Chapters 13 and 15 shows their remarkable correspondence with the objective aspects of Whitehead's actual entities. 
McHenry's third point is that "the idea that nature is alive, for Whitehead, applies at the most basic level of a universe made up of creative actualities.... Sentience applies at some level of social order and not at others (Process 103). Quantum events would not count as sentient. The societies of subatomic particles contain actual occasions with a dominance of the physical poles, but there is nothing that would give us the kind of selective choice that Malin attributes to this level of nature." I find it hard to accept this view, because if McHenry is right, this assertion would effectively deprive the actual occasions associated with elementary quantum events of any degree of creativity. Nevertheless, his reading of Whitehead may be right, in which case my view of the matter differs from Whitehead's.

The next point discussed raised by McHenry is that quantum mechanics "has the puzzling consequence of viewing the occurrence of events in nature as dependent upon our presence, and specifically our activities as physicists who perform the experiments" (Maxwell, Comprehensibility 234). Such interpretations of the formalism of quantum mechanics have been proposed, and I wish to simply distance myself from them. Such distancing clearly follows from the above dissection of the term "quantum measurement."

Bearing all of the above comments in mind, let me address the points that were raised in the next-to-last paragraph of the discussion. Consider the statement: "the rejection of realism in order to develop a more comprehensive quantum theory is not part of Whitehead's project." As I pointed out at the beginning of this response, the transcendence of realism, as I defined the term "realism," is a part of Whitehead's project. It was done, however, on philosophical grounds, not "in order to develop a more comprehensive quantum theory." To my mind, this makes the fact that Whitehead's system does fit with quantum mechanics all the more remarkable.

The rest of the paragraph calls for the following comment: grouping Einstein and Whitehead together as realists ignores significant differences between their positions. Einstein was a realist in the sense of my definition of "realism." He endorsed realism in this sense. He also endorsed locality. Since, as I showed in Chapter 7 of the book, local realism has to go, Einstein's position is now untenable. Whitehead endorsed realism in a different sense. He also endorsed locality. Now, it is a consequence of Bell's theorem that locality (as it refers to "influences," not to "signals") has to go, regardless of what happens to realism. Hence Whitehead's system has to be updated.

To summarize my response to McHenry's discussion: Part I of the book leads to the conclusion that quantum mechanics forces us to abandon local realism. This, in turn, leads to the question, what will take its place? I believe that I have shown in the book that one possible and even plausible answer to this question is "an updated version of Whitehead's theory." I believe that I have shown that the Whiteheadian approach is uniquely appropriate as a metaphysical system that can encompass the findings of quantum physics. 


\section{Works Cited}

Mailin, Shimon. Nature Loves to Hide: Quantum Physics and the Nature of Reality, a Western Perspective. New York: Oxford UP, 2001.

Maxwell, Nicholas. The Comprehensibility of the Universe: A New Conception of Science. Oxford: Clarendon Press, 1998.

Shimony, Abner. Search for a Naturalistic World View, Vol. II. Cambridge, Mass: Cambridge UP, 1993.

Whitehead, Alfred North. Process and Reality: An Essay in Cosmology. 1929. Corrected Edition. Ed. David Ray Griffin and Donald W. Sherburne. New York: Free Press, 1978. 